Aurélien Dinh

Robert Carlier

Alexis Descatha

\section{Critical illness myopathy and whole body MRI}

Received: 7 September 2015

Accepted: 12 September 2015

Published online: 21 September 2015

(C) Springer-Verlag Berlin Heidelberg and ESICM 2015

\section{A. Dinh $(\bowtie)$}

Infectious Disease Department, Teaching Hospital R. Poincaré, University Hospital of Paris, Versailles St Quentin University,

104 Bd R. Poincaré, 92380 Garches, France

e-mail: aurelien.dinh@aphp.fr

Tel.: + 33147104432

\section{R. Carlier}

Radiological Unit, Teaching Hospital R. Poincaré, University Hospital of Paris, Versailles St Quentin University, 92380 Garches, France

\section{A. Descatha}

Occupational Health Department, Teaching Hospital R. Poincaré, University Hospital of Paris, Versailles St Quentin University,

92380 Garches, France

A 36-year-old woman, hospitalized 2 months before for a right cerebral abscess with hemiplegia (neglected sinusitis) and ventilator-associated pneumonia, suddenly became tetraplegic and painful. Cerebral and medullar MRI ruled out a vascular or infectious etiology. Considering the associated pain, a whole body MRI was decided upon and revealed critical illness myopathy, confirmed by electromyography and muscle biopsy. Frontal reconstructed views on STIR sequence demonstrated an abnormal diffuse hypersignal of edematous muscles (Fig. 1a). Edema was localized in muscles with preservation of low signal of fat (fat suppression) in subcutaneous tissues and bone marrow. The outcome for the patient was progressively favorable with antalgic therapy and physiotherapy (Fig. 1b), and normalization of signal after 6 months (similar windows). Thus, MRI could be an interesting and non-invasive alternative
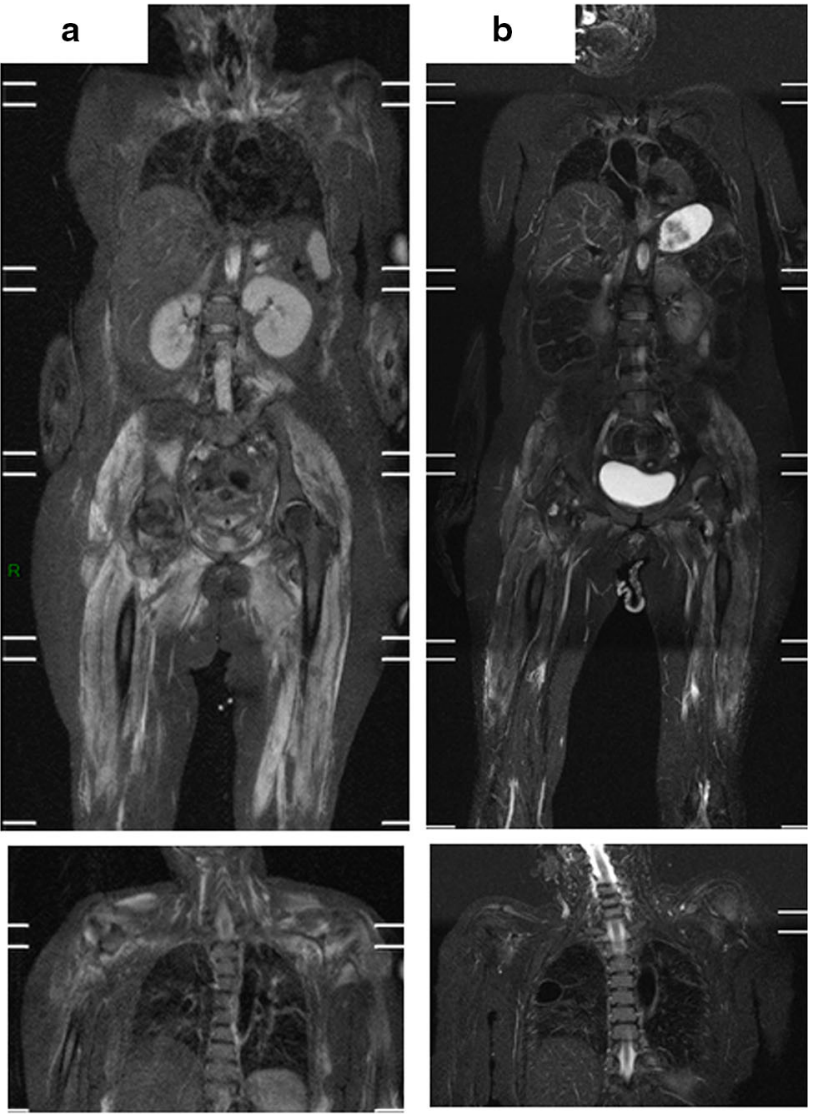

Fig. 1 a Whole body coronal reconstruction in STIR, scapular muscles below. It showed diffuse bright signal of edematous of all muscles and b normalization of signal after 6 months (similar windows)

\section{Compliance with ethical standards}

Conflicts of interest On behalf of all authors, the corresponding author states that there is no conflict of interest. 